PROCEEDINGS OF THE

AMERICAN MATHEMATICAL SOCIETY

Volume 135, Number 11, November 2007, Pages 3607-3611

S 0002-9939(07)09016-8

Article electronically published on July 2, 2007

\title{
ON A LITTLEWOOD-PALEY TYPE INEQUALITY
}

\author{
OLIVERA DJORDJEVIĆ AND MIROSLAV PAVLOVIĆ
}

(Communicated by Michael T. Lacey)

\begin{abstract}
The following is proved: If $u$ is a function harmonic in the unit ball $B \subset \mathbb{R}^{N}$ and if $0<p \leq 1$, then the inequality

$$
\int_{\partial B} u^{*}(y)^{p} d \sigma \leq C_{p, N}\left(|u(0)|^{p}+\int_{B}(1-|x|)^{p-1}|\nabla u(x)|^{p} d V(x)\right)
$$

holds, where $u^{*}$ is the nontangential maximal function of $u$. This improves a recent result of Stoll. This inequality holds for polyharmonic and hyperbolically harmonic functions as well.
\end{abstract}

Let $\mathbb{R}^{N}(N \geq 2)$ denote the $N$-dimensional Euclidean space. In [18, Stević proved that if $u$ is a function harmonic in the unit ball $B \subset \mathbb{R}^{N}$ and if $\frac{N-2}{N-1} \leq p<1$, then the inequality

$$
\sup _{0<r<1} M_{p}^{p}(r, u) \leq C_{1}|u(0)|^{p}+C_{2} \int_{B}(1-|x|)^{p-1}|\nabla u(x)|^{p} d V(x)
$$

holds. Here $d V$ denotes the Lebesgue measure in $\mathbb{R}^{N}$ normalized so that $V(B)=1$, and as usual

$$
M_{p}^{p}(r, u)=\int_{\partial B}|u(r y)|^{p} d \sigma(y)
$$

where $d \sigma$ is the normalized surface measure on the sphere $\partial B$. The strange condition $(N-2) /(N-1) \leq p \leq 1$ appears in [18] because the proof in that paper is based on the fact, due Stein and Weiss [17, 16, that $|\nabla u|^{p}$ is subharmonic for $p \geq$ $(N-2) /(N-1)$.

In the case $N=2$, inequality (11) was proved by Flett [2]. It holds for $1<p<2$ as well, while if $p>2$, then the reverse inequality holds; these inequalities are due to Littlewood and Paley 7 . Elementary proofs of the Littlewood-Paley inequalities are given in [13] and [8, 15] $(p>2)$.

In a recent paper [19, Stoll proved a very general theorem which says, in particular, that (11) holds for every $p \in(0,1]$. Here we improve Stoll's theorem by proving the following result. Here $u^{*}$ denotes the nontangential maximal function of $u$, i.e.

$$
u^{*}(y)=\sup _{|x-y|<c(1-|x|)}|u(x)|, \quad y \in \partial B,
$$

where $c>1$ is a constant.

Received by the editors August 18, 2006.

2000 Mathematics Subject Classification. Primary 31B05.

Key words and phrases. Littlewood-Paley inequalities, harmonic functions in $\mathbb{R}^{N}$. 
Theorem 1. If $u$ is a function harmonic in $B$ and if $0<p \leq 1$, then the inequality

$$
\int_{\partial B} u^{*}(y)^{p} d \sigma(y) \leq C_{p, N}\left(|u(0)|^{p}+\int_{B}(1-|x|)^{p-1}|\nabla u(x)|^{p} d V(x)\right)
$$

holds, where $C$ is a constant depending only on $p, c$, and $N$.

A well known theorem of Fefferman and Stein [1] enables us to replace $u^{*}$ in (2) by the radial maximal function $u^{+}$,

$$
u^{+}(y)=\sup _{0<r<1}|u(r y)|, \quad y \in \partial B .
$$

Namely,

Theorem A. If $U \geq 0$ is a function subharmonic in $B$ and if $p>0$, then there is a constant $C=C_{p, N, c}$ such that

$$
\int_{\partial B} U^{*}(y)^{p} d \sigma(y) \leq C \int_{\partial B} U^{+}(y)^{p} d \sigma(y) .
$$

The proof of this theorem (see [3, Theorem 3.6]), as well our proof of Theorem 11. is based on a fundamental result of Hardy and Littlewood 4 and Fefferman and Stein [1] on the subharmonic behavior of $|u|^{p}$. We state this result in the following way.

Lemma A. If $U \geq 0$ is a function subharmonic in $B(a, 2 \varepsilon)\left(a \in \mathbb{R}^{N}, \varepsilon>0\right)$, then the inequality

$$
\sup _{x \in B(a, \varepsilon)} U(x)^{p} \leq C \varepsilon^{-N} \int_{B(a, 2 \varepsilon)} U^{p} d V
$$

holds, where $C$ depends only on $p$ and $N$.

Here $B(a, r)$ denotes the ball of radius $r$ centered at $a$.

For simple proofs of Lemma $\mathrm{A}$ we refer to 10, 14, and for generalizations to various classes of functions, we refer to [5, 6, 9, 11, 12.

Let $P(x, y)$ denote the Poisson kernel,

$$
P(x, y)=\frac{1-|x|^{2}}{|x-y|^{N}} .
$$

Since $\int_{\partial B} P(x, y) d \sigma(y)=1$, we see that Theorem 1 is a direct consequence of Theorem $\mathrm{A}$ and the following:

Proposition 1. If $u$ is a function harmonic in $B$ and if $0<p \leq 1$, then the inequality

$$
u^{+}(y)^{p} \leq C_{p, N}\left(|u(0)|^{p}+\int_{B}(1-|x|)^{p-1}|\nabla u(x)|^{p} P(x, y) d V(x)\right), \quad|y|=1,
$$

holds, where $C$ is a constant depending only on $p$ and $N$.

Let

$$
u^{+}(\rho y)=\sup _{0<r<\rho}|u(r y)|=\sup _{0<t<1}|u(t \rho y)|, \quad 0<\rho \leq 1, y \in \partial B,
$$

and let $u^{+}(0)=|u(0)|$. 
Lemma 1. Let $r_{j}=1-2^{-j}$ for $j \geq 0$. If $0<p \leq 1$ and if $u$ is of class $C^{1}(B)$, then the inequality

$$
u^{+}(y)^{p} \leq C|u(0)|^{p}+C \sum_{j=0}^{\infty} 2^{-j p} \sup _{r_{j}<r<r_{j+1}}|\nabla u(r y)|^{p}, \quad y \in \partial B
$$

holds, where $C$ depends only on $p$ and $N$.

Proof. We start from the inequality

$$
u^{+}\left(r_{j+1} y\right)^{p}-u^{+}\left(r_{j} y\right)^{p} \leq \sup _{0<t<1}\left|u\left(t r_{j+1} y\right)-u\left(t r_{j} y\right)\right|^{p} .
$$

By Lagrange's theorem,

$$
\begin{aligned}
\left|u\left(t r_{j+1} y\right)-u\left(t r_{j} y\right)\right| & \leq\left(r_{j+1}-r_{j}\right) \sup _{0<r<r_{j+1}}|\nabla u(r y)| \\
& \leq 2^{-j} \sup _{0<r<r_{j+1}}|\nabla u(r y)|,
\end{aligned}
$$

and hence

$$
u^{+}\left(r_{j+1} y\right)^{p}-u^{+}\left(r_{j} y\right)^{p} \leq 2^{-j p} \sup _{0<r<r_{j+1}}|\nabla u(r y)|^{p},
$$

which implies

$$
\begin{aligned}
u^{+}(y)^{p}-|u(0)|^{p} & =\sum_{j=0}^{\infty}\left(u^{+}\left(r_{j+1} y\right)^{p}-u^{+}\left(r_{j} y\right)^{p}\right) \\
& \leq \sum_{j=0}^{\infty} 2^{-j p} \sup _{0<r<r_{j+1}}|\nabla u(r y)|^{p} .
\end{aligned}
$$

On the other hand, by summation by parts we see that if $\left\{A_{j}\right\}_{0}^{\infty}$ is a nondecreasing sequence of real numbers, then

$$
\sum_{j=0}^{\infty} 2^{-j p} A_{j+1} \leq C A_{0}+C \sum_{j=0}^{\infty} 2^{-j p}\left(A_{j+1}-A_{j}\right),
$$

where $C$ depends only on $p$. By taking $A_{j}=\sup _{0 \leq r \leq r_{j}}|\nabla u(r y)|^{p}$ and using the inequalities

$$
A_{j+1}-A_{j} \leq \sup _{r_{j}<r<r_{j+1}}|\nabla u(r y)|^{p},
$$

we get the desired result.

Proof of Proposition 1. By Lemma A with $U=|\nabla u|, a=a_{j}:=\left(r_{j}+r_{j+1}\right) y / 2$ and $\varepsilon=\left(r_{j+1}-r_{j}\right) / 2=2^{-j-2}$,

$$
2^{-j p} \sup _{r_{j}<r<r_{j+1}}|\nabla u(r y)|^{p} \leq C 2^{-j p} 2^{j N} \int_{B\left(a_{j}, 2^{-j-1}\right)}|\nabla u(x)|^{p} d V(x) .
$$

On the other hand, simple calculation shows that $\left|x-a_{j}\right| \leq 2^{-j-1}$ implies

$$
2^{-j-2} \leq 1-|x|, \quad|x-y| \leq 2^{-j+1} .
$$

Hence

$$
2^{-j} 2^{j N} \leq 2^{N+2} P(x, y), \quad \text { for } x \in B\left(a_{j}, 2^{-j-1}\right) .
$$


From this and (6) we get

$$
\begin{aligned}
2^{-j p} \sup _{r_{j}<r<r_{j+1}}|\nabla u(r y)|^{p} & \leq C 2^{-j(p-1)} \int_{r_{j-1} \leq|x| \leq r_{j+2}} P(x, y)|\nabla u(x)|^{p} d V(x) \\
& \leq 2^{1-p} C \int_{r_{j-1} \leq|x| \leq r_{j+2}}(1-|x|)^{p-1} P(x, y)|\nabla u(x)|^{p} d V(x)
\end{aligned}
$$

$\left(r_{-1}=0\right)$ where we have used the inclusion

$$
\left\{x:\left|x-a_{j}\right| \leq 2^{-j-1}\right\} \subset\left\{x: r_{j-1} \leq|x| \leq r_{j+2}\right\} .
$$

Now the desired conclusion is easily obtained by using Lemma 1 .

\section{Remarks.}

Remark 1. As the above proofs show, the validity of Proposition 1 depends only on the inequality

$$
\sup _{x \in B(a, \varepsilon)}|\nabla u(x)|^{p} \leq C \varepsilon^{-N} \int_{B(a, 2 \varepsilon)}|\nabla u|^{p} d V .
$$

It was proved in [10 that (7) is implied by

$$
|\nabla u(x)| \leq K \varepsilon^{-1} \sup _{z \in B(a, \varepsilon)}|u(z)-u(x)| .
$$

More precisely: Let $u \in C^{1}(B)$ and let $0<p<\infty$. If there is a constant $K$ such that (8) holds whenever $B(x, \varepsilon) \subset B$, then there is a constant $C=C_{p, N, K}$ such that (77) holds whenever $B(x, 2 \varepsilon) \subset B$.

Remark 2. Condition (8), and hence (7), is satisfied in a wide class of functions containing, in particular, polyharmonic, hyperbolically harmonic, and convex functions [11. Recall that $u$ is called polyharmonic if $\Delta^{k} u \equiv 0$ for some integer $k \geq 1$, and $u$ is hyperbolically harmonic if

$$
\Delta_{h} u(x):=\left(1-|x|^{2}\right)^{2}\left[\Delta u(x)+2(N-2)\left(1-|x|^{2}\right)^{-1} x \cdot \nabla u(x)\right] \equiv 0 .
$$

Note also that the class of hyperbolically harmonic functions is invariant under Möbius transformations of the ball.

Remark 3. The proof of Theorem A given in [3] (see also [14, theorem 7.1.8]) shows that (3) is implied by (4). On the other hand, as was proved in [10, condition (8) implies (4).

It follows from the above remarks that the following generalization of Theorem 1 holds.

Theorem 2. If $u$ is a function polyharmonic, hyperbolically harmonic, or convex in $B$ and if $0<p \leq 1$, then inequality (1) holds.

\section{REFERENCES}

1. C. Fefferman and E. M. Stein, $H^{p}$ spaces of several variables, Acta Math. 129 (1972), 137-193. MR 0447953 (56:6263)

2. T. M. Flett, Mean values of power series, Pacific J. Math. 25(1968), 463-494. MR0229807 $(37: 5373)$

3. J. B. Garnett, Bounded analytic functions, Pure and Applied Mathematics, 96. Academic Press, Inc. [Harcourt Brace Jovanovich, Publishers], New York-London, 1981. MR628971 (83g:30037) 
4. G. H. Hardy and J. E. Littlewood, Some properties of conjugate functions, J. Reine Angew. Math. 167 (1932), 405-423.

5. M. Jevtić, M. Pavlović, M-Besov p-classes and Hankel operators in the Bergman spaces on the unit ball, Arch. Math. 61(1993), 367-376. MR.1236315 (94i:47042)

6. M. Jevtić, M. Pavlović, On subharmonic behaviour of functions and their tangential derivatives on the unit ball in $\mathbf{C}^{n}$, Indian J. Pure Appl. Math. 30(1999), no. 4, 407-418. MR1695693 (2000c:32022)

7. J.E. Littlewood and R.E.A.C. Paley, Theorems on Fourier series and power series. II, Proc. Lond. Math. Soc. 42(1936), 52-89.

8. D.H. Luecking, A new proof of an inequality of Littlewood and Paley, Proc. Amer. Math. Soc. 103(1988), 887-893. MR947675 (89g:30067)

9. M. Pavlović, Inequalities for the gradient of eigenfunctions of the invariant Laplacian in the unit ball, Indag. Math. (N.S.) 2(1991), no. 1, 89-98. MR.1104834(92d:32008)

10. M. Pavlović, On subharmonic behaviour and oscillation of functions on balls in $\mathbf{R}^{n}$, Publ. Inst. Math. (Beograd) (N.S.) 55(69) (1994), 18-22. MR.1324970 (96d:31002)

11. M. Pavlović, Subharmonic behaviour of smooth functions, Mat. Vesnik 48 (1996), no. 1-2, 15-21. MR1410667 (97j:31007)

12. M. Pavlović, Decompositions of $L^{p}$ and Hardy spaces of polyharmonic functions, J. Math. Anal. Appl. 216(1997), 499-509. MR1489593 (99b:46029)

13. M. Pavlović, A Littlewood-Paley theorem for subharmonic functions with subharmonic Laplacian, Publ. Inst. Math. (Belgrade) 68(82)(2000), 77-82. MR1826098 (2002b:30040)

14. M. Pavlović, Introduction to function spaces on the disk, Posebna izdanja 20, Matematički Institut SANU, 2004. MR2109650 (2006d:30001)

15. M. Pavlović, A short proof of an inequality of Littlewood and Paley, Proc. Amer. Math. Soc. 134(2006), 3625-3627. MR2240675 (2007c:46030)

16. E. Stein, Singular integrals and differentiability properties of functions, Princeton University Press, Princeton, New Jersey, 1970. MR0290095 (44:7280)

17. E. M. Stein, G. Weiss, On the theory of harmonic functions of several variables. I. The theory of $H^{p}$-spaces, Acta Math. 103(1960), 25-62. MR0121579 (22:12315)

18. S. Stević, A Littlewood-Paley type inequality, Bull. Braz. Math. Soc. (N.S.) 34(2003), no. 2, 211-217. MR1992637 (2004f:31003)

19. M. Stoll, On generalizations of the Littlewood-Paley inequalities to domains in $\mathbb{R}^{n}$, IWPT (2004)

Fakultet organizacionih nauka, Jove Ilića 154, Belgrade, Serbia

E-mail address: oliveradj@fon.bg.ac.yu

Matematički fakultet, Studentski trg 16, Belgrade, Serbia

E-mail address: pavlovic@matf.bg.ac.yu 